\title{
School Dropout of Immigrant Students in the European School Systems: a Pedagogic Perspective
}

\section{ALIA \\ La dispersione scolastica degli alunni immigrati nei sistemi di istruzione europei: una lettura pedagogica}

Serena Sani

\begin{abstract}
In recent years, many scholars have highlighted persistent difficulties faced by the European school systems to contest school dropout of immigrant students (dropouts, delays, grade retention, school evasion etc.). As noted by the Council of Europe, with regard to immigrant students the average rate of school dropout in the UE is double that native pupils. Considering this situation, the objective of the paper is, on the one hand, to identify the factors that contributed to increase a similar failure and, on the other hand, to understand how Europe is trying to reduce this phenomenon. In particular, my attempt is to explain what are the educational measures and strategies adopted by UE school systems not only to clash this phenomenon, but also to achieve the ambitious target, set by the European Commission in 2015, for reducing to below $10 \%$ the percentage of school dropout by 2020 .
\end{abstract}

Keywords: school dropout, immigrant students, Europe, school policies, integration, language skills.

\section{La dispersione scolastica degli alunni immigrati: i fattori che determinano l'insuccesso scolastico}

I numerosi studi condotti negli ultimi anni a livello internazionale in merito al significativo tasso di dispersione scolastica (abbandoni, ritardi, ripetenze, evasione ecc.) nelle scuole europee, mostrano chiaramente quanto ancora il diritto all'istruzione, pur essendo ribadito ed auspicato da più parti, non sia ancora considerato nella pratica scolastica come un principio da difendere e da riconoscere a tutti gli studenti.

A conferma di ciò basti pensare che, ad oggi, in Europa, il tasso di abbandono dell'istruzione o della formazione si colloca all'11,1\% ed è

${ }^{1}$ Dipartimento di Scienze Umanistiche, Sociali e della Formazione. Università degli Studi del Molise. 
significativamente influenzato dalla situazione socio-economica delle famiglie e dal livello di istruzione dei genitori (Commissione Europea, 2015)2 .

A fronte di una simile situazione, non si può non rilevare che, nonostante gli innegabili sforzi compiuti dagli Stati membri per combattere la dispersione scolastica, i risultati sono ancora decisamente critici, poiché, attualmente, solo pochi paesi sono riusciti a sviluppare delle valide strategie di contrasto e delle adeguate azioni di prevenzione, di intervento e di compensazione.

Al riguardo, peraltro, secondo i recenti dati emersi dal monitoraggio effettuato dalla Commissione Europea nel settore dell'istruzione e della formazione presentato nella Relazione pubblicata dalla stessa nel 2015, si evince chiaramente una progressiva riduzione degli investimenti destinati all'istruzione che rischia di vanificare i progressi raggiunti finora e suscita seri dubbi sulla possibilità di perseguire l'obiettivo europeo di ridurre al di sotto del $10 \%$, entro il 2020, la percentuale di coloro che abbandonano precocemente i percorsi di istruzione e formazione (Commissione Europea, 2015) ${ }^{3}$.

In questo contesto, già di per sé alquanto problematico, si rileva un altro dato molto significativo, ossia: «il tasso medio di abbandono nell'Unione è per gli immigrati il doppio di quello degli studenti nativi» (Consiglio d'Europa, 2011, p. 2).

Secondo quanto rilevato da alcune ricerche condotte a livello internazionale $(\mathrm{OCSE}, 2007)^{4}$, tale disparità è dovuta al fatto che il livello d'istruzione della maggior parte degli alunni immigrati tende ad essere considerevolmente

\footnotetext{
${ }^{2}$ Per affrontare il fenomeno in ambito europeo si utilizza l'indicatore degli early school leavers (ESL) con il quale si prende come riferimento la quota dei giovani dai 18 ai 24 anni d'età in possesso della sola licenza media e che sono fuori dal sistema nazionale di istruzione e da quello regionale di istruzione e formazione professionale. Al riguardo si vedano, Raccomandazione del Consiglio del 28 giugno 2011 sulle politiche di riduzione dell'abbandono scolastico, in G.U. dell'Unione europea C 191, del 1.7.2011, p. 1; Camera dei deputati - VII Commissione, Presentazione degli atti dell'indagine conoscitiva sulle Strategie per contrastare la dispersione scolastica, 27 novembre 2014, www.aniat.org (Ultima rilevazione: 15/12/16).

${ }^{3}$ Secondo le stime effettuate dalla Commissione Europea, gli Stati nei quali si è registrato un taglio della spesa per l'istruzione per almeno tre anni consecutivi sono i Paesi Bassi, la Finlandia, il Portogallo, l'Italia, la Spagna, l'Irlanda e il Regno Unito. Al riguardo, si veda Commissione Europea, Monitoraggio del settore dell'istruzione e della formazione 2015, in http://ec.europa.eu/dgs/education_culture/repository/education/tools/docs/2015/monitor2015italy_it.pdf (Ultima rilevazione: $15 / 12 / 2016$ ).

${ }^{4}$ In particolare, ci riferiamo ai risultati del programma PISA (Programme for International Student Assessment) pubblicati in OCSE, Uno sguardo sull'educazione. Gli indicatori OCSE 2007, Armando Editore, Roma, 2007 e alle valutazioni del Rapporto NESSE network of experts, Education and migration strategies for integrating migrant children in European schools and societies. A synthesis of research findings for policy-makers, An independent report submitted to the European Commission by the NESSE network of experts, in http:// www.nesse.fr/nesse/activities/reports/activities/reports/education-and-migration-pdf (Ultima rilevazione: $18 / 12 / 16)$.
} 
inferiore rispetto a quello dei loro coetanei autoctoni ${ }^{5}$. Come conseguenza, ciò comporta una maggiore percentuale di casi di abbandono scolastico, livelli più bassi di qualifiche ed una parte minore di giovani che accedono all'istruzione superiore.

Rispetto ai loro compagni autoctoni, inoltre, gli alunni stranieri sono meno scolarizzati nel segmento dell'educazione pre-scolastica e in quello dell'istruzione secondaria superiore. In merito a questo ultimo grado di scuola, peraltro, vale la pena di rilevare che gli studenti immigrati sono sovrarappresentati negli istituti di istruzione professionale, i quali non sempre consentono di acquisire una preparazione adeguata per accedere agli studi universitari.

Indubbiamente, i fattori che contribuiscono a provocare l'insuccesso scolastico degli alunni provenienti da un contesto migratorio sono molteplici. Alcuni sono legati alla situazione personale: si pensi ad esempio al contesto socio-economico sfavorevole dal quale spesso provengono, alla perdita di valore delle conoscenze che hanno accumulato nel paese di origine (in particolare della lingua d'origine, ma anche delle conoscenze concernenti il funzionamento delle varie istituzioni e, nello specifico, della scuola); al mancato o ridotto riconoscimento delle qualifiche acquisite precedentemente; alle modeste aspettative delle famiglie e delle comunità; infine, all'assenza di modelli riconosciuti ai quali fare riferimento (Commissione delle Comunità Europee, 2008, p. 8).

Per quel che concerne la lingua, sappiamo bene quanto una buona conoscenza della lingua del paese ospitante rappresenti un fattore essenziale ai fini di una effettiva integrazione e di una positiva riuscita scolastica. Questa, infatti, oltre a rappresentare un valido mezzo di comunicazione nella realtà quotidiana si caratterizza come un'importante risorsa ai fini della formazione e dell'inserimento nel mercato del lavoro. Soprattutto, come sostiene il sociologo tedesco Hartmut Esser, la conoscenza della lingua e degli accenti del paese ospitante consente agli immigrati di sentirsi parte integrante del nuovo paese (Esser, 2006).

Fondamentali ai fini di una buona riuscita scolastica si rivelano le misure e le strategie educative adottate dai vari sistemi scolastici. Su questo versante, infatti, molti studi hanno rilevato che anche in situazioni simili dal punto di vista migratorio, i risultati degli alunni immigrati provenienti dai medesimi

${ }^{5}$ Per un quadro complessivo circa la questione della dispersione scolastica in Europa, cfr. K. De Witte, I. Nicaise, J. Lavrijsen, G. Van Landeghem, C. Lamote, J. Van Damme, The Impact of Institutional Context, Education and Labour Market Policies on Early School Leaving: A Comparative Analysis of EU Countries, Tier Working Paper Series Tier WP 14/12, in http://www.tierweb.nl/assets/files/UM/Workingpapers/TIERWP14-12.pdf (Ultima rilevazione: 18/12/16). 
paesi di origine variano a seconda dello Stato membro. Sostanzialmente, dunque, tali risultati inducono a pensare che l'impostazione dei sistemi d'istruzione e l'atteggiamento con il quale le scuole e gli insegnanti si rapportano agli allievi migranti possono avere un impatto decisamente rilevante sugli esiti di questi studenti (Commissione delle Comunità Europee, 2008, p. 9).

La scuola del paese ospitante, dunque, deve innanzi tutto promuovere l'integrazione scolastica di questi alunni. In questo senso, alcuni studi evidenziano come l'assenza o la presentazione distorta degli immigrati nei programmi scolastici, nei libri di testo e, in generale, nella vita scolastica può influenzare negativamente il successo scolastico di questi alunni, danneggiare la loro immagine e l'autostima di sé stessi (NESSE network of experts, 2008).

Un altro fattore che può compromettere seriamente la riuscita scolastica è l'alta concentrazione di alunni immigrati nelle stesse classi o scuole. Spesso, infatti, questi sono «concentrati in scuole che sono di fatto isolate rispetto al resto del sistema e la cui qualità tende a scadere rapidamente, come dimostra ad esempio il forte tasso di rotazione degli insegnanti» (Commissione delle Comunità Europee, 2008, p. 9) .

Tale tendenza, presente in molti sistemi europei, produce una segregazione forzata che, in molti casi, porta a risultati scolastici inferiori rispetto alla norma ed inibisce l'incremento dell'integrazione sociale, dei rapporti amicali e di una effettiva interazione tra i figli di migranti ed i loro coetanei autoctoni.

Su questo versante, vale la pena di rilevare che, spesso, anche le modalità di concentrazione o di orientamento (tracking) degli alunni secondo le loro capacità $\mathrm{o}$, comunque, inclinazioni, condizionano le future scelte di molti studenti immigrati verso quei corsi di studio che richiedono un minore impegno scolastico ed una limitata capacità dialettica (Commissione delle Comunità Europee, 2008, pp. 9-10)7.

Al riguardo, diversi studi hanno posto in luce le criticità di una simile concentrazione ed hanno rilevato che molti alunni stranieri ottengono risultati migliori quando sono inseriti in classi eterogenee con compagni che padroneggiano la lingua del paese ospitante e sono maggiormente motivati sul piano scolastico (Commissione delle Comunità Europee, 2008, p. 10).

Indubbiamente, la scuola, in quanto primo contesto di socializzazione al di fuori dell'ambito familiare, ha un ruolo molto importante nell'integrazione

\footnotetext{
${ }^{6} \mathrm{Al}$ riguardo si veda anche NESSE network of experts, Education and migration strategies for integrating migrant children in European schools and societies. A synthesis of research findings for policy-makers, cit., p. 8.

${ }^{7}$ In merito ai problemi che può provocare la segregazione scolastica, si veda anche J.S. Coleman, E.Q. Campbell, C.J. Hobson, J. McPartland, A.M. Mood, F.D. Weinfeld, R.L. York, Equality of educational opportunity, U.S. Government Printing Office, Washington, 1966, in http://files.eric.ed.gov/fulltext/ED012275.pdf (Ultima rilevazione: 28/12/16).
} 
degli alunni immigrati, per cui, «se invece di contribuire ad attenuare gli effetti dell'origine socioeconomica delle famiglie dei migranti, la scuola rifiuta, discrimina o segrega, difficilmente il processo d'integrazione avrà un esito positivo e il costo di questo insuccesso ricadrà su tutta la società» (Comitato Economico e Sociale Europeo, 2009).

Allo scopo di favorire il processo di integrazione e il successo scolastico degli alunni immigrati il CESE (Comitato Economico e Sociale Europeo) ha suggerito nel 2009 alcuni interventi molto significativi. In particolare, ha consigliato di evitare la segregazione di questi alunni in scuole frequentate soprattutto o esclusivamente dai figli degli immigrati. La scuola infatti non deve diventare un ghetto, bensì deve rispecchiare la composizione sociale della comunità e deve avere ben presente che tale segregazione in genere è affiancata o deriva da una segregazione residenziale.

Per quel che concerne gli insegnanti, il CESE ha proposto di motivare maggiormente i professori, in quanto principali protagonisti del processo educativo, non solo incentivandoli sia economicamente sia a livello di carriera, ma anche prevedendo una formazione iniziale e continua che offra loro le giuste competenze e gli adeguati strumenti per poter affrontare al meglio queste nuove realtà (Comitato Economico e Sociale Europeo, 2009, p. 85) ${ }^{8}$. $\mathrm{Su}$ tale versante, inoltre, ha consigliato di puntare anche su un incremento del numero di docenti di origine etnica e culturale diversa da quella del paese ospitante.

Per quanto riguarda la conoscenza della lingua nazionale, il CESE ha posto in evidenza l'effettiva confusione esistente tra la capacità di comunicazione e le reali conoscenze linguistiche e, a questo riguardo, ha suggerito il ricorso a sostegni linguistici specifici. Inoltre, ha indicato una serie di interventi di carattere più generale, quali: la creazione di percorsi scolastici di integrazione sin dalla prima infanzia; la promozione di forme organiche ed efficaci di interazione tra la scuola e la famiglia; il varo di corsi per l'apprendimento della lingua nazionale destinati anche ai genitori degli alunni immigrati; la presenza costante in aula di personale di supporto e di mediatori culturali (Ibidem, p. 85).

Di particolare rilievo, a nostro avviso, è l'analisi fatta dal CESE in ordine alle possibili strategie da mettere in atto per affrontare la questione dell'integrazione scolastica, a questo proposito, il Comitato dopo aver posto l'enfasi sulla necessità di promuovere una scuola inclusiva volta a correggere le mancanze di un contesto sociale sempre più caratterizzato da un irrigidimento delle politiche migratorie e, più in generale, da un acuirsi delle disparità tra

${ }^{8}$ A questo proposito, cfr. anche il parere del Comitato Economico e Sociale Europeo, Migliorare la qualità della formazione degli insegnanti, CESE 75/2008 del 16 gennaio 2008, in G.U. della Comunità Europea C 151 del 17/6/2008. 
ricchi e poveri e dal conseguente aumento dell'emarginazione sociale di tante persone in condizioni socioeconomiche svantaggiate, sottolineava l'esigenza di offrire agli alunni immigrati delle valide opportunità educative (Ibidem, p. 85) e, rivolgendosi agli Stati membri dell'Unione Europea, li esortava a rispettare l'impegno di integrazione nei riguardi degli immigrati e, ad assicurare a tutti i bambini e ai giovani, indipendentemente dalla loro posizione sociale, l'accesso al sistema educativo e il successo scolastico.

A questo scopo, il Comitato ha proposto di adottare una politica scolastica volta ad assicurare a tutti gli alunni l'accesso a scuole qualificate e gratuite; il rispetto per le differenze etniche, socio-culturali, economiche e di genere e per le caratteristiche delle singole comunità di immigrati («caratteristiche di cui tener conto nella elaborazione dei programmi con l'obiettivo dell'apertura interculturale degli istituti di istruzione»); la formazione continua dei docenti e il sostegno di personale qualificato in merito alla conoscenza della lingua e della cultura delle comunità presenti nella scuola.

Non è possibile, in questa sede, approfondire le molteplici proposte del CESE; non si può non rilevare, tuttavia, l'effettivo valore di talune di esse, come ad esempio nel caso di quelle concernenti: «la salvaguardia e la promozione del multilinguismo», la realizzazione «di un programma di tutoraggio nel quale gli studenti siano invitati ad unirsi a colleghi più grandi e con maggiore esperienza», la realizzazione di «una piattaforma di dialogo tra bambini immigrati e autoctoni, in quanto strumento capace di eliminare i preconcetti e di rafforzare l'integrazione»; l'istituzione di corsi di formazione per adulti, rivolti alle persone che provengono da un contesto migratorio; la promozione di competenze interculturali e l'assegnazione di borse di studio e altri aiuti finanziari per ridurre gli svantaggi economici; la revisione, infine, «del materiale scolastico in modo che gli immigrati non vengano presentati sotto una luce negativa».

I pareri della rete NESSE e del CESE e le indicazioni presenti in altri autorevoli studi sulla dispersione scolastica degli alunni immigrati sono stati fatti propri dal Consiglio d'Europa e dalla Commissione Europea e molte delle proposte avanzate in tali sedi sono state riprese e riportate nelle molteplici direttive presentate successivamente dai due organi istituzionali.

Tuttavia, a distanza di diversi anni dalla pubblicazione di tali proposte e dall'elaborazione di molte direttive ad esse concernenti, la situazione appare ancora alquanto problematica. Al riguardo, infatti, non si possono non rilevare le numerose difficoltà che ancora persistono nell'ambito dei diversi sistemi scolastici europei in merito all'integrazione e alla formazione scolastica di molti alunni immigrati. Ciò che continua a mancare, a nostro avviso è un effettivo coordinamento tra gli Stati membri volto ad avviare un reale confronto ed uno studio congiunto su tali questioni; la definizione 
di precisi indicatori e di validi criteri di riferimento, per quanto riguarda $\mathrm{i}$ programmi e le strategie scolastiche, atti ad evitare la dispersione scolastica e la discriminazione sociale, nonché, un sistematico monitoraggio ed un'attenta valutazione delle varie situazioni nazionali e delle differenti azioni intraprese per combattere l'emarginazione e la dispersione scolastica (Ibidem, p. 85).

\section{Le politiche di intervento suggerite dall'Unione Europea per combattere la di- spersione scolastica degli alunni immigrati}

Nelle numerose direttive emanate negli ultimi decenni dalle Istituzioni europee per contrastare l'insuccesso e la dispersione scolastica in Europa si possono trovare molti riferimenti alla specifica situazione degli alunni immigrati, tuttavia, in questa sede, si è preferito porre attenzione soltanto ad alcune direttive che esplicitano in maniera più diretta e precisa le tematiche oggetto della nostra trattazione.

In particolare, la nostra disamina intende concentrarsi su tre recenti documenti, i quali offrono un quadro abbastanza esauriente delle posizioni assunte dall'Unione Europea non soltanto in merito all'insuccesso scolastico degli alunni immigrati, ma anche rispetto all'altrettanto fondamentale esigenza di promuovere, attraverso la scuola e l'istruzione, l'effettiva integrazione sociale dei tanti immigrati che vivono nei paesi membri.

A questo proposito, si rivelano particolarmente interessanti le Conclusioni del Consiglio d'Europa del 26 novembre 2009 sull'istruzione dei bambini provenienti da un contesto migratorio. In tale documento il Consiglio d'Europa, oltre a porre l'accento sul fatto che «offrire ai bambini provenienti da un contesto migratorio migliori opportunità di successo scolastico può ridurre l'emarginazione, l'esclusione e l'alienazione» (Consiglio d'Europa, 2009), esortava gli Stati membri ad elaborare misure specifiche per l'apprendimento della lingua, poiché la buona conoscenza della lingua ufficiale (o di una delle lingue ufficiali) del paese ospitante rappresenta una delle condizione fondamentali per poter raggiungere il successo scolastico e l'integrazione sociale e professionale.

Il Consiglio d'Europa proponeva, inoltre, di intervenire con azioni mirate come «l'insegnamento intensivo della lingua per gli alunni immigrati di recente, il sostegno supplementare per quelli con difficoltà, e corsi speciali per tutti i docenti al fine di prepararli a insegnare a bambini la cui madrelingua è diversa dalla lingua d'insegnamento». Per compensare lo svantaggio in materia d'istruzione e i risultati negativi di una carente integrazione, il Consiglio d'Europa suggeriva, inoltre, di ricorrere a supporti mirati, ad esempio prevedendo maggiori risorse didattiche per le scuole collocate in zone 
svantaggiate e un'istruzione più personalizzata; nonché di offrire dei sostegni supplementari di orientamento sia agli alunni sia ai loro genitori in modo da permettere loro di poter usufruire nel modo migliore dei vari servizi ed ausili messi a disposizione dalla scuola per agevolare il percorso formativo e didattico dei discenti.

Su questo versante, peraltro, il Consiglio d'Europa sosteneva la necessità di intervenire tempestivamente e con misure mirate sin dall'arrivo dei minori in età scolare nel paese ospitante, di prevedere programmi di lunga durata per il supporto all'apprendimento linguistico e di rivedere, infine, i metodi d'insegnamento, il materiale didattico e i programmi di studi per adeguarli alle esigenze di tutti gli alunni, indipendentemente dalla loro provenienza (Consiglio d'Europa, 2009, p. 5).

Esortava altresì gli Stati membri ad individuare i principali fattori che portano all'abbandono scolastico e ad osservare le caratteristiche del fenomeno a livello nazionale, regionale e locale in modo da porre le basi per l'adozione di misure specifiche e funzionali, fondate su dati concreti.

Su un piano più generale - ma altrettanto importante ai fini della nostra disamina, poiché affronta, tra le altre cose, il problema della segregazione scolastica degli alunni immigrati e pone l'accento sulla necessità di valorizzare le differenze linguistiche e culturali, suggerendo delle valide politiche di intervento per prevenire l'insuccesso scolastico degli alunni immigrati - si pone la Raccomandazione del Consiglio d'Europa del 28 giugno 2011 sulle politiche di riduzione dell'abbandono scolastico. In questo documento, i paesi membri sono invitati ad attuare delle strategie globali incentrate su «misure di prevenzione, misure di intervento e misure di compensazione, queste ultime volte a riavvicinare allo studio coloro che hanno abbandonato l'istruzione» (Consiglio d'Europa, 2011, p. 3).

Le "misure di prevenzione", secondo le valutazioni del Consiglio d'Europa, dovrebbero avere lo scopo di prevenire i rischi di insuccesso o di abbandono scolastico attraverso una ottimizzazione dell'offerta formativa. In sostanza, l'obiettivo dovrebbe essere quello di creare delle basi solide che consentano agli alunni di sviluppare il loro potenziale e di integrarsi nella scuola. Concretamente, ciò si dovrebbe tradurre: a) nella fruizione di «un'istruzione e di un'assistenza di alta qualità fin dalla prima infanzia»; b) nell'ampliamento dell'offerta formativa, assicurando maggiori opportunità di istruzione e di formazione anche oltre l'età dell'obbligo scolastico; c) nella promozione di politiche di integrazione volte a combattere la segregazione $\mathrm{e}$ a sostenere le scuole situate in zone più svantaggiate o con molti alunni provenienti da contesti socio-economici più deboli; d) nella valorizzazione della diversità linguistica, ad esempio, aiutando i bambini immigrati a progredire nella conoscenza della lingua del paese ospitante e, ove se ne riconoscesse la necessità, anche della lingua d'origine e gli insegnanti a perfezionare 
le loro conoscenze linguistiche; e) nell'incoraggiare la partecipazione e la collaborazione dei genitori in merito alle attività scolastiche; f) nel rendere i percorsi formativi più flessibili e permeabili alle esigenze dei discenti; g) nel rendere, infine, i percorsi di formazione professionale più qualitativi, più attrattivi e maggiormente flessibili (Ibidem, p. 5).

Per quel che riguarda le "politiche di intervento", queste, secondo quanto espresso dal Consiglio d'Europa, dovrebbero combattere l'abbandono scolastico migliorando la qualità dell'istruzione e della formazione scolastica ed educativa, affrontando precocemente i problemi e fornendo un sostegno mirato agli studenti o ai gruppi di studenti a rischio di abbandono scolastico. Tali politiche devono interessare tutti i livelli dell'istruzione: dalla scuola dell'infanzia al secondo ciclo dell'istruzione secondaria. Sostanzialmente, il loro obiettivo dovrebbe essere quello di favorire l'apprendimento, di accrescere la qualità dell'insegnamento e l'innovazione pedagogica, di ampliare le competenze degli insegnanti attraverso la formazione iniziale e l'aggiornamento continuo e di fornire loro gli strumenti necessari per affrontare la diversità culturale e saper gestire le situazioni difficili.

Il Consiglio d'Europa, peraltro, riteneva particolarmente utile e importante «l'azione di mediatori appartenenti alla comunità locale, in grado di facilitare la comunicazione e ridurre la diffidenza» (Ibidem, pp. 5-6).

Nel novero delle "politiche di intervento" sono previste anche delle misure individuali di sostegno atte a fornire agli alunni delle forme di aiuto personalizzate come, ad esempio, il mentoraggio (individualmente) o il tutoraggio (in piccoli gruppi); nonché attività di orientamento e di consulenza per facilitare le scelte scolastiche o professionali.

Per quel che concerne le "politiche di compensazione", considerate fondamentali per coloro che abbandonano la scuola prima del tempo e volte a dare a tutti l'opportunità di riavvicinarsi allo studio, di frequentare corsi di istruzione e formazione e di acquisire le qualifiche che non si sono potute ottenere in precedenza, il Consiglio d'Europa proponeva di mettere a punto dei programmi d'istruzione della "seconda opportunità".

Tali programmi, dovrebbero offrire un apprendimento specifico, corrispondente alle esigenze di coloro che hanno abbandonato la scuola, e devono essere adottati con piccoli gruppi di studenti, all'interno di un insegnamento personalizzato, innovativo e adeguato all'età, caratterizzato da percorsi formativi flessibili.

Sempre nell'ambito delle "politiche di compensazione", veniva proposto di istituire dei corsi di recupero volti a facilitare il reinserimento nel sistema generale di istruzione e a colmare le comprensibili lacune formatesi con l'interruzione precoce degli studi; di prevedere, inoltre, delle misure di riconoscimento e di convalida della formazione precedente (Consiglio d'Europa, 2011, p. 6). 
Un terzo documento che, a nostro avviso, vale la pena di esaminare è la Raccomandazione del Comitato dei Ministri agli Stati membri sull'importanza delle competenze nella(e) lingua(e) di scolarizzazione per l'equità e la qualità nell'istruzione e per il successo scolastico (Consiglio d'Europa, 2014). In tale documento, pubblicato nel 2014, il Comitato dei Ministri, pur non riferendosi esclusivamente ai problemi linguistici degli alunni immigrati, offre dei suggerimenti molto utili in merito alla necessità di impartire a tutti gli alunni adeguate competenze nella lingua di scolarizzazione.

$\mathrm{Al}$ riguardo, partendo dall'assunto che il diritto all'istruzione non può essere completamente esercitato se coloro che devono apprendere «non padroneggiano le norme linguistiche proprie della scuola e necessarie per avere accesso alla conoscenza», il Comitato dei Ministri rilevava come la mancanza di competenze linguistiche condizionasse notevolmente il successo scolastico e creasse serie condizioni di svantaggio e di diseguaglianza tra gli alunni.

Per ovviare a questi problemi, i responsabili dei sistemi scolastici, devono operare per garantire a tutti i discenti, l'acquisizione, non soltanto delle competenze necessarie alla comunicazione ordinaria, ma anche di quelle della lingua più «accademica» utilizzata nell'insegnamento delle varie discipline.

In generale, gli estensori del documento auspicavano una maggiore sensibilità da parte del personale educativo nel valutare le diverse lingue presenti nella scuola come una risorsa, ed esortavano gli insegnanti e i responsabili del settore a valutare periodicamente, soprattutto al momento del passaggio da un livello scolastico a quello successivo, le effettive capacità degli alunni «di padroneggiare le forme della lingua di scolarizzazione richieste ai diversi livelli scolastici, in modo da adattare la progressione degli insegnamenti e predisporre le forme di sostegno adeguate che tengano conto dei bisogni e delle capacità specifiche degli apprendenti» (Ibidem, p. 8).

Su questo versante, la scuola è chiamata ad attivarsi per offrire, in generale a tutti gli allievi, la qualità e l'equità nell'istruzione e, in particolare per gli alunni di lingua minoritaria o immigrati, un'attenzione particolare alle loro esigenze e alle loro effettive capacità linguistiche (Ibidem, p. 11).

Il Comitato dei Ministri ricordava, infine, alcune iniziative attuate dal Consiglio d'Europa per promuovere la cooperazione a livello europeo, quali: l'organizzazione di forum di scambio per i responsabili dell'istruzione dei vari Stati membri; la raccolta e la presentazione - a cura dell'Unità delle politiche linguistiche, sul sito Internet del Consiglio d'Europa, nello specifico

\footnotetext{
${ }^{9}$ La traduzione della Raccomandazione $C M / \operatorname{Rec}(2014) 5$ e della relativa appendice utilizzata in questa sede è a cura di Edoardo Lugarini ed è pubblicata da "Italiano LinguaDue" per concessione del Council of Europe. Al riguardo si veda, Università degli Studi di Milano, "Italiano LinguaDue", 2014, in www.italianolinguadue.unimi.it (Ultima rilevazione: 10/01/2017).
} 
sulla Piattaforma delle risorse e dei riferimenti per un'educazione plurilingue ed interculturale -, dei risultati di esperienze positive; l'assistenza agli Stati membri, con l'Unità delle politiche linguistiche, nello sviluppo di curricoli scolastici allo scopo di specificare chiaramente le competenze linguistiche necessarie per insegnare ed apprendere tutte le materie scolastiche; l'accordo con lo European Centre for Modern Languages (ECML) per la formazione di formatori degli insegnanti e del personale amministrativo della scuola sulla rilevanza linguistica di ogni processo di insegnamento e di apprendimento (Ibidem, pp. 8-9).

Tali iniziative, a nostro avviso, sono di vitale importanza, poiché da un lato consentono, come si è già detto, di promuovere un reale confronto ed un'effettiva collaborazione a livello europeo, dall'altro indicano chiaramente la volontà di sensibilizzare e responsabilizzare coloro che, a vario titolo, occupano un ruolo importante nel settore dell'istruzione e dell'educazione in merito alla necessità di garantire a tutti gli alunni in generale e a quelli immigrati in particolare l'acquisizione di adeguate competenze linguistiche necessarie per raggiungere un buon successo scolastico.

In questa prospettiva si comprende bene come, la più volte ribadita esigenza di creare condizioni di equità per tutti gli studenti, a prescindere dal loro status socio-economico, debba fare i conti con la consapevolezza della sfida che il possesso delle competenze nella lingua di scolarizzazione rappresenta per il successo scolastico.

\section{Le azioni intraprese dagli Stati europei per contrastare la dispersione scola- stica degli alunni immigrati}

In molti paesi europei l'offerta di un supporto linguistico agli studenti immigrati - relativo non soltanto all'apprendimento della lingua del paese ospitante, ma anche al mantenimento della lingua di origine - è già molto presente nei percorsi formativi.

In Danimarca, Svezia, Slovenia e Svizzera, ad esempio, gli studenti hanno la possibilità di frequentare lezioni nella propria lingua madre, oltre a quelle di recupero nella lingua in cui viene impartito l'insegnamento. In Lussemburgo questo tipo di offerta è attiva in alcune scuole primarie per gli studenti di madrelingua portoghese. L'obiettivo di queste lezioni è quello di aiutare gli alunni a mantenere e a sviluppare le capacità linguistiche nella lingua madre e offrire loro le basi per apprendere altre lingue. In molti Länder tedeschi, invece, all'inizio del ciclo dell'istruzione primaria e secondaria tutti gli studenti vengono valutati nella lingua in cui viene impartito l'insegnamento e i risultati di tale valutazione costituiscono la base per sviluppare programmi linguistici 
su misura per le esigenze specifiche degli studenti. Diversi altri paesi, tra i quali Malta e Austria, hanno inserito nuove politiche o misure per potenziare il sostegno linguistico allo scopo di ridurre i tassi di abbandono scolastico precoce. In paesi come Belgio (Comunità francese), Grecia e Cipro ciò si traduce nella possibilità, per gli studenti appena arrivati, di passare un tempo più lungo nelle classi di accoglienza (Commissione europea/EACEA/Eurydice/ Cedefop, 2014, pp. 60-61).

Come abbiamo visto, tuttavia, la mancanza di competenze linguistiche è solo uno dei fattori che possono portare all'insuccesso scolastico degli alunni immigrati, poiché spesso giocano a loro sfavore anche le barriere culturali, la segregazione socio-economica, la carenza di valide misure di sostegno per favorire l'apprendimento etc. Su questo versante, un altro aspetto da considerare è che, secondo quanto rilevato dal Rapporto Eurydice e Cedefop del 2014, i fattori che provocano tale dispersione non sono gli stessi per tutti gli Stati membri.

$\mathrm{Al}$ riguardo, peraltro, dal Rapporto Eurydice e Cedefop emerge chiaramente che in merito a tale questione, $\mathrm{i}$ dati relativi ai bambini o agli adolescenti immigrati inseriti nei sistemi scolastici europei presentano notevoli lacune. In particolare, le informazioni sull'abbandono precoce degli studenti nati all'estero fornite dalle autorità statistiche nazionali a Eurostat in molti casi risultano poco affidabili, mentre per taluni paesi i dati più recenti non sono disponibili a causa dell'esiguità del campione. In generale, i dati sui giovani nati all'estero che abbandonano precocemente la scuola spesso risultano essere «inaccurati, senza parlare dei casi di immigrazioni non registrate/irregolari, impossibili dunque da quantificare. Infine, a livello dell'UE non esistono dati confrontabili per i migranti di seconda generazione» (Ibidem, p. 9).

Secondo quanto riportato nel Rapporto Eurydice e Cedefop, la provenienza da un contesto di immigrazione o da una minoranza, di per sé, non dovrebbe essere considerata un fattore determinante in relazione all'abbandono precoce. In questo senso, infatti, si rileva che i fattori che esercitano un'influenza maggiore sul percorso scolastico e che incidono maggiormente in caso di abbandono precoce, sono quelli legati alle condizioni socio-economiche degli studenti. In particolare, si ritiene che «situazioni familiari difficili come disoccupazione, basso reddito del nucleo familiare e scarsi livelli di istruzione dei genitori possono avere un effetto diretto e duraturo sulla carriera scolastica degli studenti, sul loro atteggiamento nei confronti dello studio, sui loro risultati scolastici e, di conseguenza, ciò può indurli a decidere di abbandonare precocemente i percorsi di istruzione e formazione» (Ibidem, p. 9) ${ }^{10}$.

${ }^{10}$ Per un ulteriore approfondimento in merito ai fattori che, in generale, possono provocare l'abbandono scolastico, cfr. R. Thibert, Le décrochage scolaire: diversité des approches, 
Al riguardo, tuttavia, non si può negare che, se è vero che la provenienza da un contesto di immigrazione di per sé non può essere considerata un fattore determinante è pur vero che spesso sono proprio le famiglie immigrate a risultare più colpite dallo svantaggio socio-economico rispetto alle famiglie autoctone e questo potrebbe far chiarezza sui risultati più modesti e, talvolta più negativi degli alunni immigrati (Commissione Europea, 2013).

La progressiva consapevolezza dei problemi che può causare l'abbandono precoce della scuola, non soltanto per i diretti interessati, ma anche per la società, ha indotto l'Unione Europea e gli Stati membri ad impegnarsi a ridurre la percentuale di giovani che abbandonano precocemente gli studi e la formazione al di sotto del 10\% entro il 2020 (Council of the European Union, 2009).

Alla luce di tale decisione, anche i Ministri dell'Istruzione dell'U.E. hanno ritenuto opportuno accordarsi su un «quadro di riferimento per politiche coerenti, complete e basate su dati certi» per contrastare l'abbandono precoce ${ }^{11}$. A tale scopo, sono stati istituiti dei gruppi di lavoro composti da decisori politici e operatori del settore di tutta Europa con l'obiettivo di favorire lo sviluppo delle politiche dei vari Stati membri attraverso l'apprendimento reciproco e l'individuazione di buone pratiche ${ }^{12}$.

In questo contesto, deve essere inserita anche l'elaborazione del già citato Rapporto Eurydice e Cedefop del 2014 sulla Lotta all'abbandono precoce dei percorsi di istruzione e formazione in Europa: strategie, politiche e misure. Tale documento, infatti, mira a rafforzare l'impegno in questo ambito sia della Commissione Europea sia degli Stati membri e si occupa di monitorare gli sviluppi in merito alla progettazione e all'attuazione di strategie globali, incentrate su dati certi, per la lotta all'abbandono precoce e il sostegno all'apprendimento degli studenti. Sostanzialmente, il Rapporto del 2014 provvede a fornire molti dati aggiornati ed ampie indicazioni circa le politiche e le misure più recenti attuate dai paesi europei per favorire la riduzione dell'abbandono precoce dei percorsi di istruzione e formazione (Commissione europea/EACEA/Eurydice/Cedefop, 2014, pp. 17-18).

Per quel che concerne i dati sugli studenti immigrati che abbandonano la scuola prima del tempo, vale la pena di rilevare che i tassi riguardanti

diversité des dispositifs, Dossier d'actualité - Veille et Analyses, 84, Mai 2013, Lyon: Institut Français de l'Éducation, in: http://ife.ens-lyon.fr/vst/DA-Veille/84-mai-2013 (Ultima rilevazione: 10/01/17).

${ }^{11}$ Cfr. la già citata Raccomandazione del Consiglio del 28 giugno 2011 sulle politiche di riduzione dell'abbandono scolastico, in G.U. dell'Unione Europea C 191 del 1/7/2011.

${ }^{12}$ Per un approfondimento sul ruolo e sugli obiettivi dei gruppi di lavoro, si veda European Commission, Education and Training 2020 ET 2020 Working Groups 2016-2018, in http:// ec.europa.eu/education/policy/strategic-framework/expert-groups_en.htm (Ultima rilevazione: 15/01/2017) 
l'abbandono precoce, in media, sono del $\ll 22,6 \%$ tra i nati all'estero contro $1 ' 11 \%$ della popolazione studentesca nativa».

In paesi come Danimarca, Irlanda, Malta, Paesi Bassi e Portogallo le differenze tra i due gruppi risultano relativamente esigue, anche se in alcuni di questi paesi si registrano alti tassi di migranti. Infine, vi è un paese che costituisce un'eccezione: si tratta del Regno Unito, che infatti rileva una percentuale di studenti che abbandonano precocemente i percorsi di istruzione e formazione lievemente più alto tra gli autoctoni (Ibidem, p. 39).

Secondo uno studio condotto in Spagna, gli studenti di origine straniera hanno il $16 \%$ di probabilità in più rispetto agli autoctoni, di abbandonare precocemente l'istruzione (IVIE, 2013). A conferma di ciò, basti pensare che, nell'anno 2013, il tasso di ELET (Abbandono precoce dei percorsi di istruzione e formazione) degli studenti stranieri è stato quasi il doppio di quello degli studenti spagnoli (Ibidem, p. 39) ${ }^{13}$.

A questo proposito, ci sembra importante accennare ad alcune ipotesi di ricerca avanzate in un interessante studio condotto nei Paesi Bassi e relativo alle minoranze etniche. In particolare, si considera degna di nota l'ipotesi secondo la quale gli studenti immigrati o appartenenti a minoranze etniche potrebbero essere maggiormente a rischio di abbandono precoce rispetto al resto della popolazione studentesca perché privati di quel "capitale umano" che fornisce un contesto di apprendimento cognitivamente stimolante per lo sviluppo del bambino. In tal senso, sono chiamati in causa anche i livelli di qualifiche scolastiche dei genitori e si rileva che più alto è il loro grado di istruzione, più basse sono le probabilità di dispersione scolastica dei figli. Infine, nell'ambito dello stesso studio, si evidenzia che nei Paesi Bassi gli immigranti di seconda generazione solitamente hanno un grado di istruzione inferiore rispetto al resto della popolazione studentesca e tale condizione può avere un impatto negativo sulle loro prospettive di impiego.

Un ultimo aspetto importante posto in rilievo da questo e da altri studi sull'argomento è che spesso i bambini che provengono da un contesto migratorio hanno minori probabilità di frequentare il ciclo di istruzione della prima infanzia, il quale, come è noto, offre un decisivo contributo allo sviluppo delle abilità linguistiche e relazionali che rappresentano dei fattori fondamentali per il successo scolastico (Traag e van der Velden, 2011, pp. 45-62).

Un altro dato importante che vale la pena di rilevare riguarda l'eterogeneità dei percorsi di formazione degli alunni immigrati. Questi, infatti, pur provenendo tutti da un contesto migratorio, presentano notevoli differenze in

13 Tali dati, tuttavia, non sembra possano «dare pienamente conto della tendenza all'abbandono precoce in Spagna, dato che la sola percentuale di studenti migranti non spiega le differenze nei tassi di ELET tra le Comunità autonome». 
termini di risultati scolastici. Al riguardo, alcuni studi hanno dimostrato che gli alunni immigrati di recente ottengono risultati decisamente inferiori rispetto a quelli di seconda generazione (Luciak, 2004).

Le condizioni socio-economiche della famiglia (occupazione, composizione del nucleo familiare, ambiente abitativo ecc.), come si è accennato in precedenza, hanno certamente la loro influenza sul successo scolastico, soprattutto a mano a mano che il bambino coglie le differenze tra la sua e le famiglie dei suoi compagni autoctoni. Non sono infatti da sottovalutare gli effetti psicologici che può provocare la progressiva consapevolezza non soltanto delle condizioni di emarginazione che vive la sua famiglia, ma anche delle difficoltà linguistiche e della scarsa posizione sociale dei suoi genitori (Commissione Europea, 2013). Tale posizione di marginalità della famiglia rende particolarmente difficoltosi i processi di identificazione e imitazione tanto importanti per un'armoniosa maturazione della propria identità e non facilita certamente il processo di allargamento dello spazio vitale e cognitivo verso l'ambiente extrafamiliare (Demetrio, Favaro, 1992, p. 60).

In ambito scolastico, la dimensione migratoria e le condizioni socioeconomiche finiscono spesso per diventare le cause principali della segregazione formativa di molti studenti immigrati. Secondo alcuni studi,

la segregazione nell'istruzione può verificarsi per diversi motivi. Da una parte, può essere determinata dalla selezione all'interno del sistema educativo. Una valutazione degli studenti che non tenga sufficientemente conto del contesto svantaggiato o delle origini migranti degli studenti potrebbe far sì che risultassero sovra-rappresentati nelle scuole 'svantaggiate' o addirittura assegnati all'educazione speciale. D'altra parte, la segregazione sociale delle scuole potrebbe dipendere dalla tendenza dei diversi gruppi sociali a vivere in aree differenti (Commissione europea/EACEA/Eurydice/Cedefop, 2014, p. 45) ${ }^{14}$.

Comunque, indipendentemente dai motivi che possono portare alla segregazione degli alunni immigrati, non si può non rilevare l'inadeguatezza di tale pratica. Diversi studi, infatti, hanno dimostrato che nelle scuole con un alto livello di segregazione si registrano risultati decisamente più negativi, si riscontrano comportamenti più problematici da parte degli studenti (Hugh, 2010, pp. 261-276) e un più alto rischio di abbandono precoce (Lyche, 2010) ${ }^{15}$.

${ }^{14}$ Al riguardo, si veda anche OCSE, No More Failures: Ten Steps to Equity in Education, Paris, OECD, 2007.

${ }_{15}$ A tale proposito, cfr. anche T. Traag e R.K.W. van der Velden, Early school-leaving in the Netherlands: the role of family resources, school composition and background characteristics in early school-leaving in lower secondary education, cit. pp. 45-62; A.M. Nevala et al., Reducing early leaving from education and training in the EU. European Parliament, 
In concreto, «l'effetto della segregazione socio-economica sulla composizione della popolazione studentesca all'interno di una scuola è così potente che persino uno studente medio è più incline alla dispersione» (Commissione europea/EACEA/Eurydice/Cedefop, 2014, p. 45).

Di per sé, anche la separazione precoce degli studenti in percorsi di formazione scolastica differenziati in base alle loro attitudini può avere delle ripercussioni negative. Per gli alunni immigrati, infatti, tale prassi si traduce, in molti casi, nell'inserimento precoce in percorsi più pratici che non consentono loro di sviluppare in maniera adeguata le competenze linguistiche, sociali e culturali necessarie per esprimere pienamente il loro potenziale e, talvolta, non corrispondono affatto alle loro attitudini e aspirazioni ${ }^{16}$.

Un simile pratica, inoltre, può contribuire ad accentuare sensibilmente le differenze e le disuguaglianze in merito ai risultati scolastici e, nei casi in cui è accompagnata da un rigido sistema scolastico che non consente un'effettiva permeabilità dei percorsi formativi, può provocare negli studenti che conseguono scarsi risultati scolastici, la perdita della motivazione a proseguire i loro studi (Commissione Europea, 2011).

La differenziazione precoce può causare «un circolo vizioso nelle aspettative di insegnanti e studenti. Gli insegnanti possono avere aspettative minori nei confronti degli studenti con scarsi risultati e di conseguenza questi adeguano le proprie aspettative e i propri sforzi. Inoltre, gli studenti cessano di trarre vantaggi dall'influenza positiva data dal fatto di essere circondati da compagni più bravi» (Commissione europea/EACEA/Eurydice/Cedefop, 2014, p. 45$)^{17}$.

Su un piano più generale, se l'appello rivolto agli Stati membri nella Raccomandazione del Consiglio del 28 giugno 2011, ad adottare delle politiche mirate ed efficaci e ad attuare una strategia globale incentrata su «misure di

Brussels, 2011, in http://www.europarl.europa.eu/activities/committees/studies/download. do?language $=$ en $\&$ file $=42311$ (Ultima rilevazione: $18 / 01 / 2017)$.

${ }^{16}$ Per un approfondimento in merito a tale pratica cfr., B. Spinath, F. Spinat, Development of self-perceived ability in elementary school: The role of parents' perceptions, teacher evaluations, and intelligence, Cognitive Development, 20, 2005, pp. 190-204; J. Hattie, Visible Learning. A Synthesis of over 800 Meta-analyses relating to achievement, Routledge, London, 2009; OCSE, Closing the Gap for Immigrant Students: Policies, Practice and Performance. Reviews of Migrant Education, OECD, Paris, 2010.

${ }^{17} \mathrm{Al}$ riguardo si vedano anche: E.A. Hanushek e L. Wößmann, Does Educational Tracking Affect Performance and Inequality? Differences-in-Differences Evidence across Countries, Economic Journal, 116 (510), 2006, pp. 63-76; R. Rumberger e S.A. Lim, Why Students Drop Out of School: A Review of 25 Years of Research, 2008, in http://www.slocounty.ca.gov/Assets/ CSN/PDF/Flyer+-+Why+students+drop+out.pdf (Ultima rilevazione: 17/01/20117); OCSE, Equity and Quality in Education: Supporting Disadvantaged Students and Schools, OECD, Paris, 2012. 
prevenzione», «misure di intervento» e «misure di compensazione» (Consiglio d'Europa, 2011, p. 3) è stato accolto da diversi paesi, nel complesso, tuttavia, in tutta Europa sono soltanto sei i paesi che ad oggi hanno sviluppato una simile strategia globale, ossia la Bulgaria, Malta, i Paesi Bassi, l'Austria, il Belgio (Comunità fiamminga) e la Spagna. In particolare, i primi quattro paesi si sono attivati proprio in seguito a tale Raccomandazione, mentre gli ultimi due avevano già provveduto in precedenza. Altre due nazioni (Ungheria e Romania) si trovano nella "fase di adozione di una strategia globale" e, in diversi altri paesi (Germania, Irlanda, Polonia, Portogallo, Regno Unito e Norvegia), benché non si possa parlare di una strategia globale nei termini sopracitati, sussistono «altre strategie o piani d'azione di ampio respiro, volti a garantire a giovani e adulti l'opportunità di completare l'istruzione e di ottenere le qualifiche di cui hanno bisogno per avere successo in ambito lavorativo» (Commissione europea/EACEA/Eurydice/Cedefop, 2014, p. 51).

Tutti gli Stati, comunque, hanno introdotto politiche e misure volte a contrastare l'abbandono anticipato o, in ogni caso, si stanno attrezzando per ridurre i tassi di abbandono precoce (Ibidem, p. 51).

In Germania, Cipro, Austria, Islanda e Norvegia, le politiche di intervento sono incentrate sull'identificazione precoce degli studenti in difficoltà e su misure di sostegno volte a favorire l'apprendimento. In Lussemburgo e in Portogallo sono previste classi speciali e/o personale docente in più per gli alunni che richiedono un sostegno supplementare all'apprendimento. A Malta, in Polonia e in Slovenia è previsto un sostegno aggiuntivo permanente, volto a soddisfare le esigenze formative e a promuovere lo sviluppo individuale degli studenti. In Belgio (Comunità francese) esistono Piani di apprendimento individuali (PIA-Plan individuel d'apprentissage) che forniscono agli studenti il sostegno necessario per poter colmare le proprie lacune nell'apprendimento e acquisire le competenze necessarie per poter terminare il loro percorso di studi. In Danimarca, il sistema scolastico affida il sostegno degli studenti con scarso rendimento a personale specializzato. In Spagna, infine, durante il normale orario delle lezioni, sono previste misure di sostegno scolastico all'interno o al di fuori della classe, da parte del personale docente. L'obiettivo è quello di migliorare l'apprendimento e i risultati scolastici, favorendo così anche l'integrazione sociale degli studenti.

Su questo versante, altri paesi, tra cui l'Italia e la Lettonia, hanno adottato delle politiche volte a favorire il potenziamento delle competenze degli insegnanti, in modo da offrire agli alunni svantaggiati un percorso scolastico focalizzato sulla persona e sulle sue esigenze. In undici paesi (Irlanda, Grecia, Spagna, Lituania, Lussemburgo, Ungheria, Malta, Slovenia, Finlandia, Islanda, Svizzera) per contrastare l'abbandono precoce sono attuate delle misure di intervento che mirano a garantire l'integrazione di tutti gli studenti con bisogni 
educativi speciali. Infine, in Bulgaria, Grecia, Spagna, Croazia, Romania, Irlanda e Slovenia per gli studenti che provengono da contesti socio-economici svantaggiati, sono previsti anche supporti di tipo finanziario, sotto forma di «libri di testo, trasporto gratuito da e per la scuola e/o borse di studio»; oppure pasti scolastici gratuiti o, ancora, «incentivi finanziari per gli studenti o le loro famiglie a patto che i giovani continuino a frequentare la scuola» (Ibidem, p. 58).

Per quel che concerne più specificamente gli studenti immigrati e quelli che provengono da minoranze etniche, molti paesi, oltre a mettere in atto misure di sostegno linguistico, mettono anche in pratica dei progetti volti a promuovere la formazione e l'integrazione di questi alunni. In Grecia, ad esempio, a partire dal 2010 il Ministero dell'istruzione, in collaborazione con alcune Università, ha realizzato diversi progetti, tra cui «Formazione professionale degli studenti immigrati e rimpatriati» e «Istruzione dei bambini della minoranza mussulmana in Tracia». L'obiettivo generale di tali progetti è quello di combattere l'insuccesso scolastico, di ridurre le percentuali di dispersione e di garantire a tutti gli studenti un'effettiva integrazione sociale e pari opportunità formative (Ibidem, p. 65).

Da questa rapida disamina sulle politiche scolastiche adottate in Europa per contrastare il fenomeno della dispersione scolastica emerge, indubbiamente, la chiara volontà sia dell'Unione Europea, sia dei singoli Stati membri di impegnarsi a risolvere questa difficile questione; ciò che emerge da recenti studi condotti sul tema, tuttavia, è che sul piano concreto, «nonostante i paesi si stiano in generale avvicinando al benchmark europeo di ridurre, entro il 2020, la percentuale di coloro che abbandonano precocemente i percorsi di istruzione e formazione al di sotto del $10 \%$, questo fenomeno continua a rimanere un serio problema per la maggioranza degli Stati membri». Fino ad ora, infatti, anche se tutti i paesi hanno elaborato varie misure per combattere l'abbandono scolastico degli alunni immigrati o comunque a rischio, solo pochi hanno sviluppato una specifica strategia di contrasto, che comprenda le auspicate misure di prevenzione, di intervento e di compensazione (Baggiani, 2015).

Per quanto riguarda l'istruzione e l'integrazione sociale e scolastica dei numerosi alunni stranieri presenti nelle scuole europee, infine, non può essere taciuto il fatto che le importanti proposte avanzate dal Consiglio e dalla Commissione e i significativi sforzi messi in atto dai vari Stati membri per ridurre l'abbandono scolastico non potranno sortire gli effetti auspicati se non si prende seriamente in considerazione la necessità di fornire al personale docente e a tutti coloro che a diverso titolo operano nella scuola, un'adeguata formazione alle competenze interculturali.

$\mathrm{Su}$ questo versante, infatti, se le scuole europee vogliono raggiungere risultati positivi in merito alla sfida posta dalla nuova realtà multietnica e 
multiculturale devono assolutamente provvedere ad incrementare e a rendere più efficienti i corsi di formazione iniziale e continua degli insegnanti (Comitato Economico e Sociale Europeo, 2009).

\section{Bibliografia}

Baggiani S., Sintesi del Rapporto di Eurydice, Tackling Early Leaving from Education and Training in Europe - Strategies, Policies and Measures, 2014, testo disponibile al sito: http://www.indire.it/eurydice/content/index.php?action=read_notizie\&id_ $\mathrm{cnt}=16662$ (Ultima rilevazione: $25 / 01 / 2017)$.

Camera dei deputati - VII Commissione, Presentazione degli atti dell'indagine conoscitiva sulle Strategie per contrastare la dispersione scolastica, 27 novembre 2014, testo disponibile al sito: www.aniat.org (Ultima rilevazione: 15/12/16).

Coleman J.S., Campbell E.Q., Hobson C.J., McPartland J., Mood A.M., Weinfeld F.D., York R.L., Equality of educational opportunity, U.S. Government Printing Office, Washington, 1966, testo disponibile al sito: http://files.eric.ed.gov/fulltext/ ED012275.pdf (Ultima rilevazione: 28/12/16).

Comitato Economico e Sociale Europeo, Migliorare la qualità della formazione degli insegnanti, CESE 75/2008 del 16 gennaio 2008, in G.U. della Comunità Europea C 151 del 17/6/2008.

Comitato Economico e Sociale Europeo, Parere del Comitato Economico e Sociale Europeo in merito al Libro verde - Migrazione e mobilità: le sfide e le opportunità per i sistemi d'istruzione europei, $\operatorname{COM}(2008) 423$ def., Bruxelles, 25 febbraio 2009, in G.U. dell'Unione Europea C 218 del 11/09/2009.

Commissione delle Comunità Europee, Libro Verde - Migrazione e mobilità: le sfide e le opportunità per i sistemi d'istruzione europei $\{\operatorname{SEC}(2008) 2173\}$ COM/2008/0423 def. Bruxelles, 3/7/2008.

Commissione Europea, Reducing early school leaving, 2011, testo disponibile al sito: http://eur-lex.europa.eu/LexUriServ/LexUriServ.do?uri=SEC:2011:0096:FIN: EN:PDF (Ultima rilevazione: 18/01/2017).

Commissione Europea, Study on Educational Support to newly arrived migrant children, 2013, testo disponibile al sito: http://bookshop.europa.eu/en/study-oneducational-support-for-newly-arrivedmigrant-children-pbNC3112385/ (Ultima rilevazione: 17/01/2017).

Commissione Europea/EACEA/Eurydice/Cedefop, Lotta all'abbandono precoce dei percorsi di istruzione e formazione in Europa: strategie, politiche e misure, Rapporto Eurydice e Cedefop, Lussemburgo: Ufficio delle pubblicazioni dell'Unione Europea, 2014, testo disponibile al sito: http://www.indire.it/lucabas/ lkmw_img/eurydice/TacklingEarlyLeavingfromEducationandTraininginEuropeStrategiesPoliciesandMeasures_IT.pdf (Ultima rilevazione: 10/01/17).

Commissione Europea, Monitoraggio del settore dell'istruzione e della formazione 2015, testo disponibile al sito: http://ec.europa.eu/dgs/education_culture/repository/ education/tools/docs/2015/monitor2015-italy_it.pdf (Ultima rilevazione: 15/12/2016). 
Council of the European Union, Council conclusions of 12 May 2009 on a strategic framework for European cooperation in education and training ('ET 2020'), Official Journal of the European Union C 119, of the 28/5/2009, testo disponibile al sito: http://eur-lex.europa.eu/legal-content/EN/TXT/PDF/?uri=CELEX:52009XG 0528(01)\&from=EN (Ultima rilevazione: 11/01/17).

Consiglio d'Europa, Conclusioni del Consiglio del 26 novembre 2009 sull'istruzione dei bambini provenienti da un contesto migratorio, in G.U. dell'Unione Europea C 301 del 11/12/2009.

Consiglio d'Europa, Raccomandazione del Consiglio del 28 giugno 2011 sulle politiche di riduzione dell'abbandono scolastico, in G.U. dell'Unione europea C 191, del 1.7.2011.

Consiglio d'Europa, Raccomandazione CM/ REC(2014)5 del Comitato dei Ministri agli Stati membri sull'importanza delle competenze nella(e) lingua(e) di scolarizzazione per l'equità e la qualità nell'istruzione e per il successo scolastico (Adottata dal Comitato dei Ministri il 2 aprile 2014 in occasione della riunione 1196 dei Delegati dei Ministri), testo disponibile al sito: https://www.coe.int/t/ dg4/linguistic/Source/Source2014/RecCM-(2014)5_LangScol_It.pdf (Ultima rilevazione: 10/01/2017).

Demetrio D., Favaro G., Immigrazione e pedagogia interculturale. Bambini, adulti, comunità nel percorso di integrazione, Firenze, La Nuova Italia, 1992.

De Witte K., Nicaise I., Lavrijsen J., Van Landeghem G., Lamote C., Van Damme J., The Impact of Institutional Context, Education and Labour Market Policies on Early School Leaving: A Comparative Analysis of EU Countries, Tier Working Paper Series Tier WP 14/12, testo disponibile al sito: http://www.tierweb.nl/assets/ files/UM/Workingpapers/TIERWP14-12.pdf (Ultima rilevazione: 18/12/16).

Esser H., Migration, sprache und integration, AKI-Forschungsbilanz 4, Arbeitsstelle Interkulturelle Konflikte und gesellschaftliche Integration (AKI) Wissenschaftszentrum Berlin für Sozialforschung (WZB), Januar 2006.

European Commission, Education and Training 2020 ET 2020 Working Groups 2016-2018, testo disponibile al sito: http://ec.europa.eu/education/policy/strategicframework/expert-groups_en.htm (Ultima rilevazione: 15/01/2017).

Hanushek E.A., Wößmann L., Does Educational Tracking Affect Performance and Inequality? Differences-in-Differences Evidence across Countries, Economic Journal, 116 (510), 2006.

Hattie J., Visible Learning. A Synthesis of over 800 Meta-analyses relating to achievement, London, Routledge, 2009.

Hugh D., The 'collateral impact' of pupil behaviour and geographically concentrated socioeconomic disadvantage, British Journal of Sociology of Education, 31, 2010.

IVIE (Instituto Valenciano de Investigaciones Económicas), El abandono educativo temprano: Análisis del caso español, 2013, testo disponibile al sito: http://www. mecd.gob.es/dctm/inee/documentos-de-trabajo/abandono-educativotemprano. pdf?documentId=0901e72b8173034a (Ultima rilevazione: 15/01/17).

Luciak M., Migrants, minorities and education - Documenting discrimination and integration in 15 member states of the European Union, Office for Office Publications of the European Communities, Luxembourg, 2004, testo disponibile 
al sito: http://fra.europa.eu/sites/default/files/fra_uploads/186-CS-Education-en.pdf (Ultima rilevazione: 15/01/2017).

Lyche C., Taking on the completion challenge: A literature review on policies to prevent dropout and early school leaving, OECD Education Working Papers, 53, 2010, testo disponibile al sito: http://dx.doi.org/10.1787/5km4m2t59cmr-en (Ultima rilevazione: 17/01/2007).

NESSE network of experts, Education and migration strategies for integrating migrant children in European schools and societies. A synthesis of research findings for policy-makers. An independent report submitted to the European Commission by the NESSE network of experts, 2008, testo disponibile al sito: http://www.nesse.fr/nesse/activities/reports/activities/reports/education-andmigration (Ultima rilevazione: 18/12/16)

Nevala A.M., Hawley J., Stokes D., Slater K., Souto Otero M., Santos R., Duchemin C., Manoudi A., Reducing early leaving from education and training in the EU. European Parliament, Brussels, 2011, testo disponibile al sito: http://www.europarl. europa.eu/activities/committees/studies/download.do?language $=$ en $\&$ file $=42311$ (Ultima rilevazione: 18/01/2017).

OCSE, Closing the Gap for Immigrant Students: Policies, Practice and Performance. Reviews of Migrant Education, Paris, OECD, 2010.

OCSE, Equity and Quality in Education: Supporting Disadvantaged Students and Schools, Paris, OECD, 2012.

PISA (Programme for International Student Assessment) pubblicati in OCSE, Uno sguardo sull'educazione. Gli indicatori OCSE 2007, Roma, Armando Editore, 2007.

Rumberger R., Lim S.A., Why Students Drop Out of School: A Review of 25 Years of Research, 2008, testo disponibile al sito: http://www.slocounty.ca.gov/Assets/CSN/ PDF/Flyer+-+Why+students+drop+out.pdf (Ultima rilevazione: 17/01/20117).

Spinath B., Spinat F., Development of self-perceived ability in elementary school: The role of parents' perceptions, teacher evaluations, and intelligence, Cognitive Development, 20, 2005.

Thibert R., Le décrochage scolaire: diversité des approches, diversité des dispositifs, Dossier d'actualité - Veille et Analyses, 84, Mai 2013, Lyon: Institut Français de l'Éducation, testo disponibile al sito: http://ife.ens-lyon.fr/vst/DA-Veille/84mai-2013 (Ultima rilevazione: 10/01/17).

Traag T., van der Velden R.K.W., Early school-leaving in the Netherlands: the role of family resources, school composition and background characteristics in early school-leaving in lower secondary education, Irish Educational Studies, 30 (1), 2011. 